\title{
LE DIACÉTYLE DANS LES BEURRES NORMANDS
}

\author{
par MM. \\ CH. 13RIOUX \\ Ingénieur agronome \\ Directeur \\ et \\ EDG. JOUIS \\ Ingénieur agricole \\ Chef de Travaux \\ de la Station agronomique de la Seine-Inférieure (1).
}

La question du diacétyle dans les beurres, actuellement à l'ordre du jour, vient d'être reprise dans la Revue "Le Lait ", par M. J. Pien et ses collaborateurs, qui en ont donné une étude très complète ( 2 et 3), et décrit dans leur dernier article une nouvelle méthode d'analyse permettant, lorsque l'on opère sur 500 grammes de beurre, de doser le diacétyle arec exactitude à partir de $0 \mathrm{mgr}$. 1 par kilogramme, ou, si l'on opère seulement sur 100 grammes, à partir de 0 mgr. 5 par kilogramme.

Cette méthode, que M. Pien a eu l'amabilité de nous communiquer avant même qu'elle ne fut publiée, nous a permis de mener à bien les recherches sur la présence du diacétyle dans les beurres normands (Calvados et Manche principalement), qui ont été demandées à la Station agronomique de la S'eine-Inférieure par le Ministère de l'Agriculture.

Nous prions done nos lecteurs de se reporter au dernier article de MM. Pien, Baisse et Martin (Le Lait, no 167, 1937, p. 673 à 698 ), pour tout ce qui concerne la question analytique.

Fn dehors du diacétyle, il nous a paru utile de doser également dans un certain nombre de crèmes et de beurres, ne fut-ce qu'à titre de documentation, l'acétylméthylearbinol, produit également et en plus grande abondance au cours des fermentations lactiques naturelles, surtout lorsqu'interviennent plus ou moins les bactéries du groupe Lactis aerogenes.

On sait que l'acétylméthylcarbinol peut, par oxydation de son groupement $\mathrm{CHOH}$, donner un peu de diacétyle, et qu'inversement le diacétyle, sous l'influence d'actions réductrices, est susceptible de se retransformer en acétylméthylcarbinol.

Pour doser ce dernier corps dans la crème, le beurre ou le babeurre, nous opérions de la façon suivante:

Un prélèvement de 100 grammes, introduit dans un ballon de 300 à $400 \mathrm{~cm}^{3}$, est additionné de $20 \mathrm{~cm}^{3}$ d'une solution de perchlorure

(1) Collaboration analytique de MHe Le CACHEUx, Préparateur.

(2) J. Pien, J. Baisse et R. Martin. Contribution à l'étude du diacétyle, Le Lait, $1936, n^{\circ} 152$ et 153.

(3) J. Pien, J. Baisse et R. Martin. Le dosage du diacétyle dans les beurres, Le Lait, juillet-août $1937, \mathrm{n}^{\circ} 167$. 
de fer officinal, et l'on traite comme pour le dosage du diacétyle, par un courant de vapeur d'eau.

L'acétylméthylcarbinol s'oxyde progressivement en donnant du diacétyle ; la distillation doit être lente ; on recueille $50 \mathrm{~cm}^{3}$ en 50 à 60 minutes.

Après avoir rendu le distillat homogène, on en prélève $10 \mathrm{~cm}^{3}$ sur lesquels on pratique la réaction colorimétrique de M. Pren; parfois on est obligé de diluer pour obtenir une teinte pas trop forte, comparable à l'échelle établie.

On obtient la somme diacétyle plus acétylméthylcarbinol, évaluée en diacétyle; il est facile d'en déduire l'acétylméthylcarbinol seul. Nous l'avons également dosé, dans quelques échantillons, par la méthode de M. Lemorgne, au diméthylglyoximate de nickel.

Prélèvement des échantillons de beurre. - Le prélèvement des échantillons devait être effectué principalement dans les départements de la Manche et du Calvados.

Après entente arec les Directeurs des Services agricoles de ces deux départements et l'Inspection régionale de la Répression des Fraudes, M. BARBAzAN, inspecteur de ce Service, effectuant normalement des tournées dans la Manche et le Calvados, voulut bien accepter de nous prêter son concours, et nous tenons à le remercier de sa bonne collaboration, et des soins qu'il apporta aux divers prélèvements et au transport des échantillons.

Les prélèvements furent, dans leur ensemble, répartis en trois catégories :

$1^{\circ}$ Beurres fermiers, prélevés sur des mottes mises en vente directement sur les marchés par les cultivateurs producteurs.

$2^{\circ}$ Beurres de commerce. Provenant de marchands en gros qui font des mélanges et remalaxent les beurres fermiers achetés sur les marchés.

$3^{\circ}$ Beurres provenant des coopératives de producteurs et de laiteriesbeurreries industrielles. Dans chaque cas, des prélèvements de 500 grammes furent effectués nous permettant de doser le diacétyle sur la totalité ou seulement sur 400 grammes, et l'acétylméthylcarbinol sur 100 grammes.

Je dois indiquer que les prélèvements eurent lieu généralement par temps frais ou modérément chaud; les échantillons, transportés en automobile, étaient apportés dans le plus bref délai à la Station agronomique par M. BARBAzAN lui-même. On les conservait à la glacière réglée à $+5^{\circ}$ ou $+6^{\circ}$, et l'analyse était généralement effectuée dans les 48 heures suivant leur réception.

-L'évolution susceptible de se produire dans le beurre, que nous 
mentionnerons à la fin de cette note, devait être peu importante ou nulle, et l'on peut considérer les chiffres cités comme se rapportant à des beurres frais, de très récente fabrication.

Résultats obtenus. - Nous donnons dans les tableaux qui suivent le détail de nos résultats, exprimant le diacétyle en milligrammes par kilogramme de beurre, et lorsqu'il y a lieu, l'acétylméthylcarbinol, évalué en $\mathrm{CH}^{3}-\mathrm{CHOH}-\mathrm{CO}-\mathrm{CH}^{3}$. On pourrait presque se dispenser de la correction, puisque $\$ 6$ de diacétyle correspondent à 88 d'acétylméthylcarbinol.

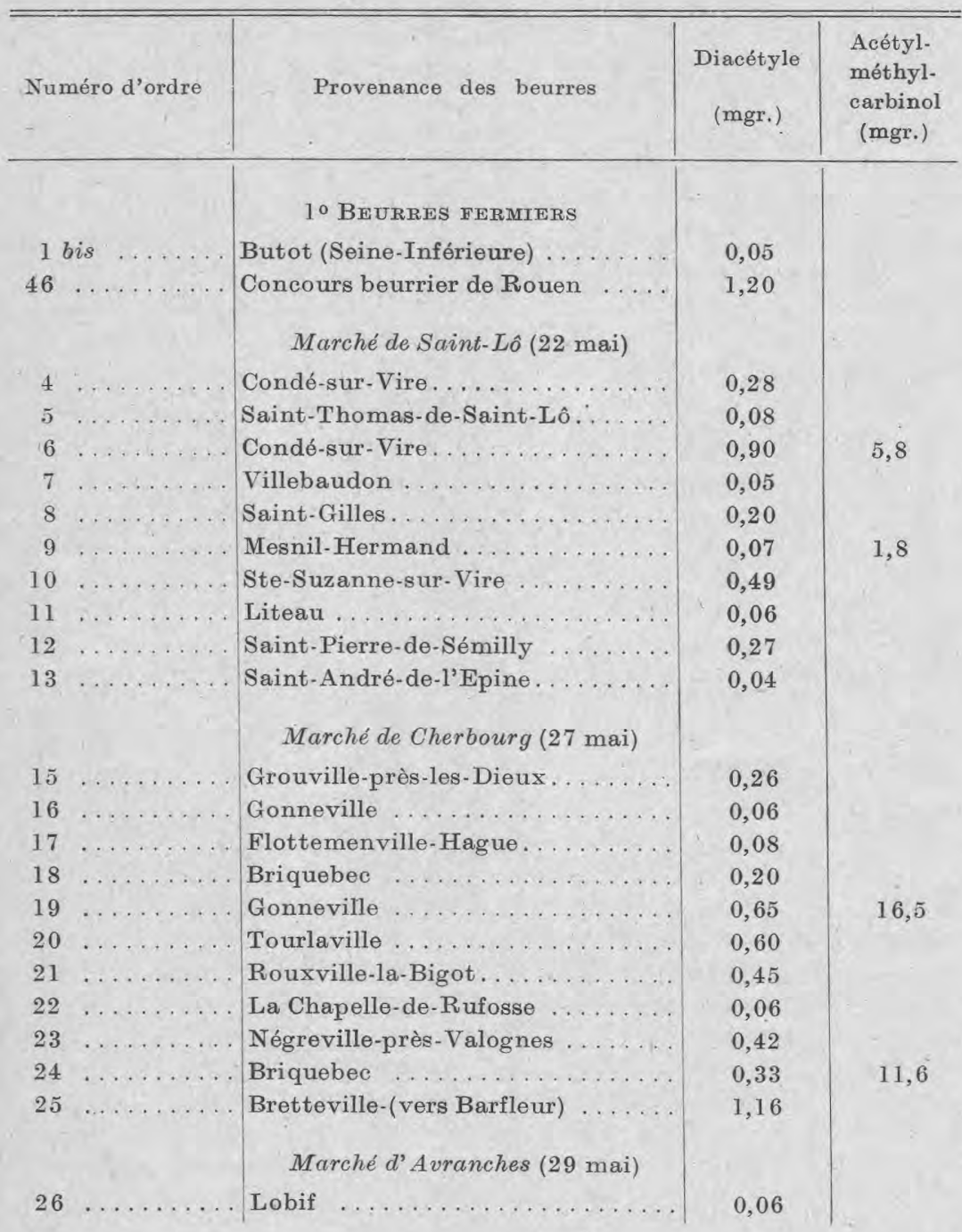




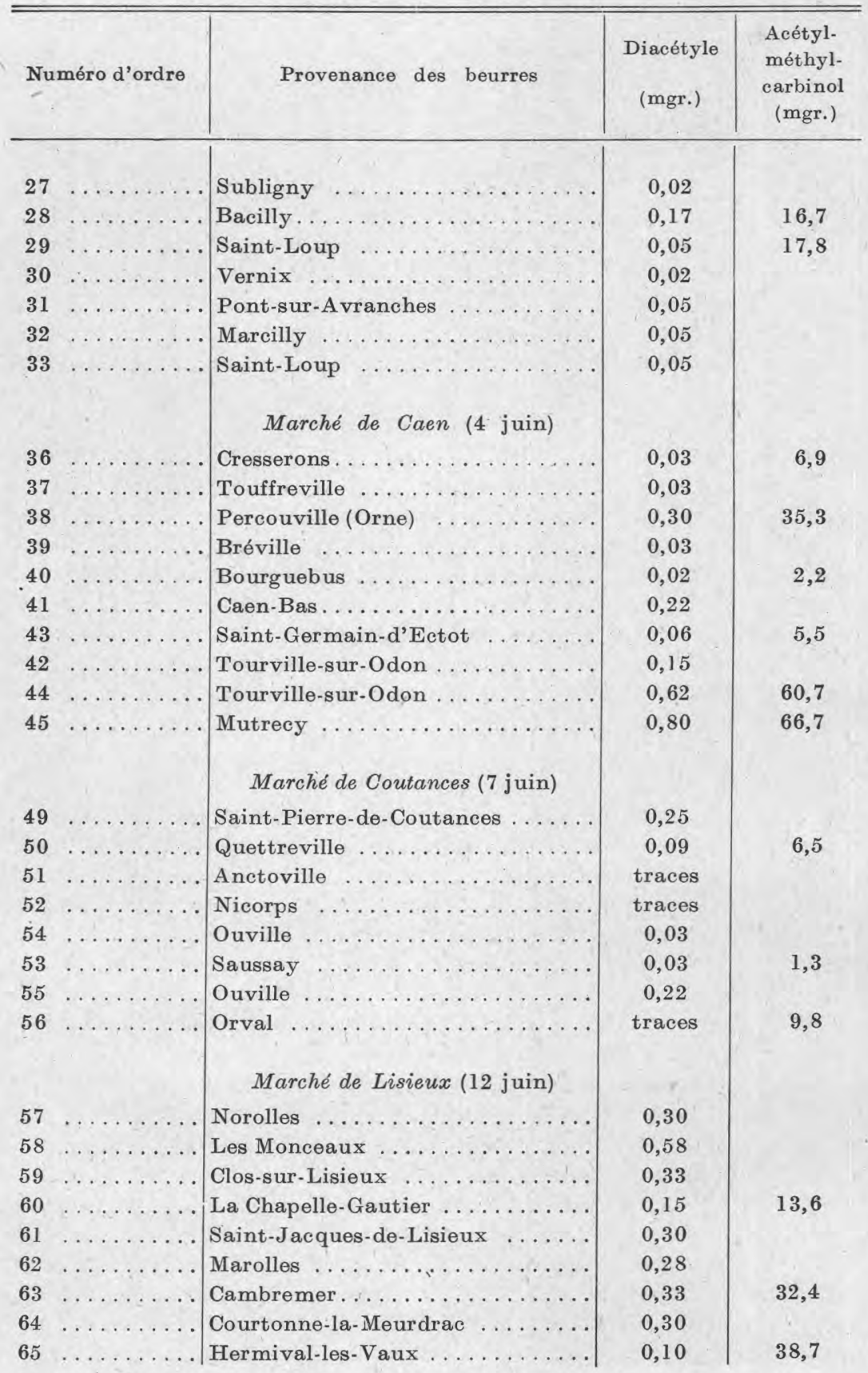




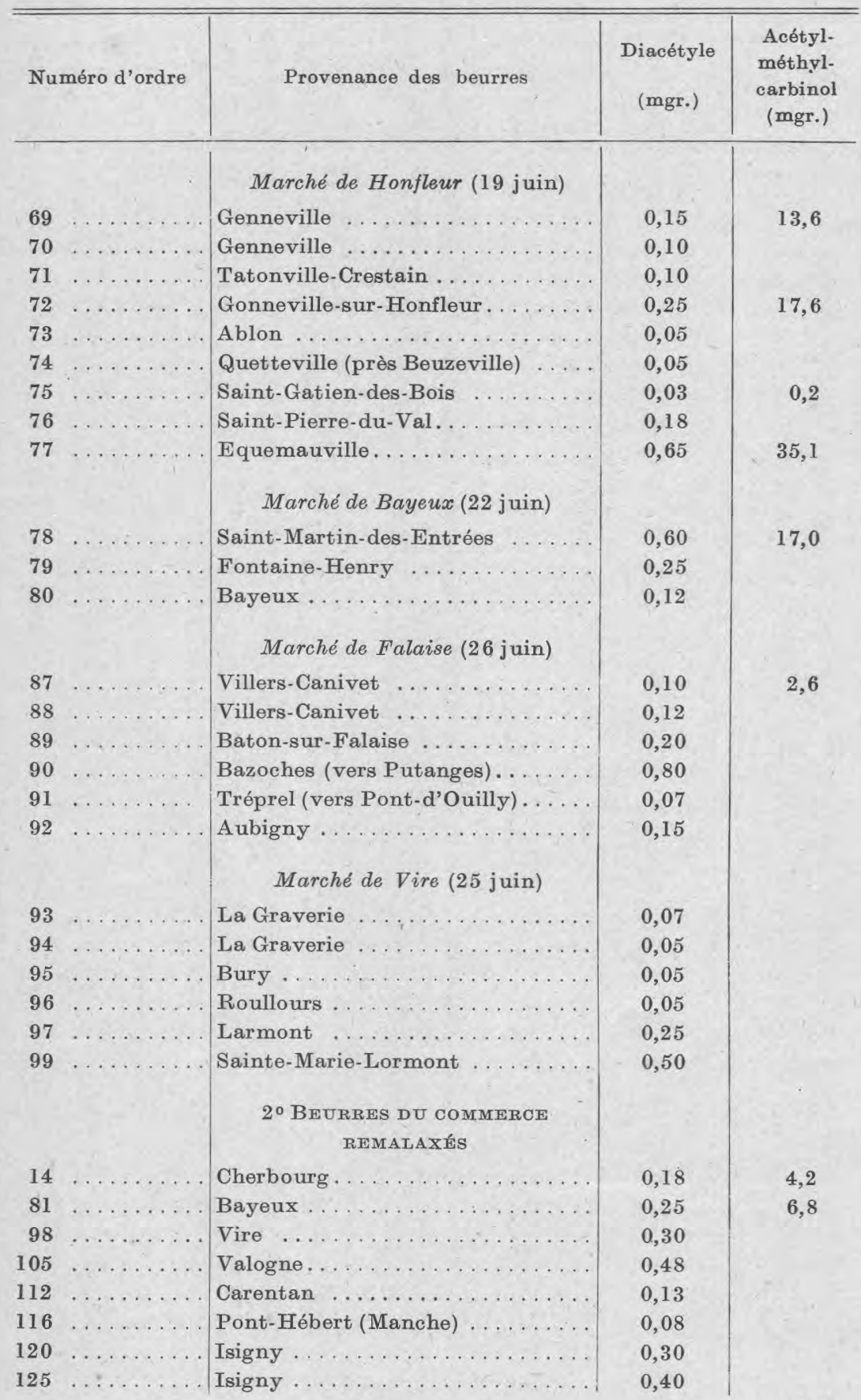




\begin{tabular}{|c|c|c|c|}
\hline Numéro d'ordre & Provenance des beurres & $\begin{array}{l}\text { Diacétyle } \\
\text { (mgr.) }\end{array}$ & $\begin{array}{l}\text { Acétyl- } \\
\text { méthyl- } \\
\text { carbinol } \\
\text { (mgr.) }\end{array}$ \\
\hline & $\begin{array}{l}\text { 30 BEURRES DES LAITERIES } \\
\text { COOPERATIVES ET INDUSTRIELLES }\end{array}$ & & \\
\hline$\ldots \ldots \ldots \ldots$ & Fermiers d'Isigny $\ldots \ldots \ldots \ldots \ldots$ & 0,30 & . \\
\hline $85 \ldots \ldots \ldots$ & Laiteries Coop. Villers-Bocage ... & 0,60 & 19,8 \\
\hline $118 \ldots \ldots \ldots \ldots$ & Laiterie Lanquetot, les Veys .... & 0,78 & \\
\hline $119 \ldots \ldots \ldots \ldots$ & Laiterie Dupont, à Isigny . . . . . & 1,13 & 40,3 \\
\hline $127 \ldots \ldots \ldots \ldots$ & $\begin{array}{r}\text { Laiterie Coop. de Bayeux, Vau- } \\
\text { celles } \ldots \ldots \ldots \ldots \ldots \ldots \ldots\end{array}$ & 0,42 & 32,1 \\
\hline$\ldots \ldots \ldots \ldots$ & Laiterie Coopérative d'Isigny .... & 0,45 & \\
\hline $129 \ldots \ldots \ldots \ldots$ & Fermiers Normands, à Bayeux ... & 0,15 & . \\
\hline $3 \ldots \ldots \ldots$ & Laiterie de Quettrèville (Manche) & 0,50 & \\
\hline $34 \ldots \ldots \ldots \ldots$ & $\begin{array}{l}\text { Fermiers du Bas-Cotentin, à Tré- } \\
\text { behou } \ldots \ldots \ldots \ldots \ldots \ldots\end{array}$ & 0,90 & 22,6 \\
\hline 48 & Laiterie Coopérative de Ducey.... & 1,15 & 26,9 \\
\hline 100 & Laiterie Coop. de Montebourg . . . . & 0,63 & \\
\hline$\ldots \ldots \ldots \ldots$ & Laiterie Coop. de Valognes ...... & 1,25 & \\
\hline$\ldots \ldots \ldots \ldots$ & Laiterie Bretel, à Valognes . . . . . & 0,90 & \\
\hline$\ldots \ldots \ldots \ldots$ & $\begin{array}{l}\text { Laiterie Coopérative de } \text { St-Sau- } \\
\text { veur-le-Vicomte } \ldots \ldots \ldots \ldots \ldots\end{array}$ & 0,90 & \\
\hline 109 & $\begin{array}{l}\text { Industrie Laitière du Cotentin, à } \\
\text { Saint-Sauveur-le-Vicomte .... }\end{array}$ & 1,28 & 22,9 \\
\hline $111 \ldots \ldots \ldots$ & Société " Lait Gloria », à Carentan & 0,15 & \\
\hline $113 \ldots \ldots \ldots \ldots$ & Laiterie Coop. de Périers......... & 0,75 & \\
\hline 115 & Laiterie Claudel, à Pont-Hébert . . & 0,82 & 15,9 \\
\hline 122 & $\begin{array}{l}\text { Laiterie Coopérative Ste-Mère- } \\
\text { Eglise, à Chef-du-Pont } \ldots . . .\end{array}$ & 2,43 & 69,1 \\
\hline$\ldots \ldots \ldots \ldots \ldots$ & $\begin{array}{l}\text { Laiterie Industrielle de Chef-du- } \\
\text { Pont } \ldots \ldots \ldots \ldots \ldots \ldots \ldots \ldots \ldots\end{array}$ & 1,32 & 22,7 \\
\hline 2 & Laiterie de Cany (Seine-Infér.).... & 0,50 & 6,6 \\
\hline$\ldots \ldots \ldots \ldots$ & $\begin{array}{l}\text { Laiterie Coopérative de la Vallée } \\
\text { de la Saône, à Royville (S.-I.) }\end{array}$ & 1,13 & 23,4 \\
\hline$\ldots \ldots \ldots \ldots$ & $\begin{array}{l}\text { Laiterie Coopérative de la Vallée } \\
\text { de la Saône, à Royville (S.-I.) }\end{array}$ & 1,50 & 42,4 \\
\hline
\end{tabular}

Il résulte de l'examen de ces tableaux que les doses de diacétyle trouvées normalement dans les beurres normands sont généralement très faibles.

Pour la totalité des échantillons de beurre, les dosages se répartissent ainsi : 


\begin{tabular}{|c|c|c|}
\hline Teneur en diacétyle & $\begin{array}{c}\text { Nombre } \\
\text { d'échantillons }\end{array}$ & Soit pour 100 \\
\hline De traces à 0 mgr. 5 par kilogramme ...... & 24 & 20,4 \\
\hline De $0 \mathrm{mgr} .06$ à $0 \mathrm{mgr} .10$ par kilogramme & 18 & 15,2 \\
\hline De $0 \mathrm{mgr} .11$ à $0 \mathrm{mgr} .50$ par kilogramme... & 48 & 40,7 \\
\hline De 0 mgr. 51 à $1 \mathrm{mgr} .00$ par kilogramme... & 18 & 15,3 \\
\hline De 1 mgr. 00 à 1 mgr. 50 par kilogramme... & 9 & 7,6 \\
\hline \multirow[t]{2}{*}{ De $1 \mathrm{mgr} .50$ à $2 \mathrm{mgr} .50$ par kilogramme } & 1 & 0,8 \\
\hline & 118 & 100,0 \\
\hline
\end{tabular}

Donc $76,3 \%$ des beurres examinés jusqu'ici contiennent des doses de diacétyle qui varient de traces à 0 mgr.5 par kilogramme.

Les beurres fermiers, achetés sur certains marchés sont partieulièrement pauvres en diacétyle, tels ceux provenant des régions d'Avranches et de Coutances, ainsi que d'une partie du rayon d'approvisionnement de Caen. On trouve cependant, pour quelques beurres fermiers, des dosages supérieurs à 0 mgr. 5 et allant jusqu'à 1 milligramme.

Les beurres remalaxés du commerce, en assez petit nombre, d'ailleurs, avaient tous une teneur en diacétyle inférieure à $0 \mathrm{mgr}$. 50 par kilogramme.

Les chiffres les plus élevés sont fournis, contrairement à ce que nous avions d'abord supposé, par les beurres des Laiteries Coopíratives ou Industrielles. Le beurre obtenu au Concours beurrier de Rouen, au début de juin, dosait lui-même $1 \mathrm{mgr}$. 2 de diacétyle, et ne peut être suspecté d'aucune addition; c'est done la nature des ferments lactiques qui doit intervenir.

Si nous faisons, pour l'ensemble des beurres des laiteries coopératives et industrielles, le même tableau résumé par teneurs croissantes, comme précédemment, nous obtenons une répartition tout à fait différente.

Bien que nos analyses n'aient porté que sur 23 beurres de laiteries, le pourcentage des forts dosages en diacétyle, e'est-à-dire de ceux dépassant $0 \mathrm{mgr}$. 50 et $1 \mathrm{mgr}$. 0 par kilogramme, est assez typique, et comme une partie de ces beurres prov́ient de crèmes stérilisées et réensemencées, il faut admettre soit une certaine prolifération de bacilles du-genre Lactis aerogenes, ou voisins, producteurs d'acétylméthylcarbinol, et d'un peu de diacétyle, soit la production de diacétyle par les ferments lactiques vrais (1).

(1) Voir les comptes-rendus du 11 e Congrès international de Laiterie de Berlin (Rapport du professenr Mohr). 
BEURRES DES LAITERIES

\begin{tabular}{c|c|c}
\hline \hline \multicolumn{1}{c|}{ Teneur en diacétyle } & $\begin{array}{c}\text { Nombre } \\
\text { d'échantillons }\end{array}$ & Soit pour 100 \\
De traces à 0 mgr. 05 par kilogramme ... & 0 & 0 \\
De 0 mgr. 06 à 0 mgr. 10 par kilogramme. & 0 & 0 \\
De 0 mgr. 11 à 0 mgr. 50 par kilogramme. & 7 & 30,4 \\
De 0 mgr. 51 à 1 mgr. 00 par kilogramme. & 8 & 34,8 \\
De 1 mgr. 00 à 1 mgr. 50 par kilogramme. & 7 & 30,4 \\
De 1 mgr. 50 à 2 mgr. 50 par kilogramme. & 1 & 4,3 \\
& - & $-19,9$ \\
\hline \hline
\end{tabular}

On aurait plutôt songé à rencontrer les plus fortes teneurs en diacétyle pour les beurres fermiers provenant de crèmes ayant subi une fermentation moins dirigée et moins soignée ; mais comme nous le verrons plus loin, il est possible que le diacétyle, formé peut-être dans les premiers stades de la fermentation lactique, et très volatil, disparaisse en partie lorsque la crème n'est barattée qu'après plusieurs jours, alors que dans les laiteries coopératives et industrielles, les crèmes sont barattées le jour qui suit l'écrémage.

Ce n'est là qu'une opinion qui pourrait être contrôlée lors de recherches effectuées directement dans un laboratoire de grande laiterie, où l'on pourrait suivre facilement l'évolution de la matière première. Je puis indiquer déjà qu'une crème de la laiterie de Royville (S.-I.), du 23 juin, analysée le 24, jour du barattage, nous a donné $1 \mathrm{mgr}$. 9 de diacétyle; le lendemain, elle n'en contenait plus que 1 mgr. 5 .

D'autre part, en ce qui concerne la relativement haute teneur en diacétyle du beurre provenant de la Laiterie Coopérative de la Société Mère-Eglise ( 2 mgr. 4 par kilogramme), beurre résultant du barattage d'une crème pasteurisée puis réensemencée à l'aide de ferments danois, nous n'avons pu jusqu'ici préciser les causes de cette teneur élevée (1); il faudrait examiner les cultures de ferments utilisées, doser le diacétyle dans les crèmes fermentées au moment du barattage, et aussitôt après dans le beurre frais et dans le babeurre avant tout lavage. Cela ne peut se faire d'une façon précise que sur place, à moins que le laboratoire ne soit que peu distant de la laiterie dont on étudie les produits.

Nous verrons plus loin les résultats que nous avons trouvés,

(1) Un autre échantillon de beurre provenant de la même laiterie nous a donné 1 mgr. 6 de diacétyle par $\mathrm{kg}$, avec $3 \mathrm{mgr}$. 5 dans le babeurre. Le ferment utilisé dosait $0 \mathrm{mgr} .4$ de diacétyle par $\mathrm{kg}$. 
gräce à la bonne collaboration de M. Floutier, directeur de la Laiterie Coopérative de la vallée de la Saâne à Royville (S.-I.).

Teneur des beurres en acétylméthylcarbinol. - Nous n'avons dosé l'acétylméthylcarbinol que dans le tiers environ des beurres analysés, en choisissant alternativement des beurres pauvres, moyennement riches ou riches en diacétyle, de façon à voir s'il existe une corrélation quelconque entre la teneur des beurres en ces deux éléments.

L'examen du tableau montre de suite que les beurres renferment en général notablement plus d'acétylméthylcarbinol que de diacétyle. Les chiffres trouvés varient de $1 \mathrm{mgr}$. 3 à $69 \mathrm{mgr}$. par kilogramme et se répartissent de la façon suivante.

\begin{tabular}{|c|c|c|}
\hline Teneurs en acótylméthylearbinol & $\begin{array}{c}\text { Nombre } \\
\text { d'échantillons }\end{array}$ & Soit pour 100 \\
\hline De 1 mgr. 3 à 10 mgr. par kilogramme & 17 & $37,8), 72$, \\
\hline De $10 \mathrm{mgr} .0$ à $25 \mathrm{mgr}$. par kilogramme & 16 & $35,5\}$ \\
\hline De $25 \mathrm{mgr} .0$ à $50 \mathrm{mgr}$. par kilogramme & 9 & 20,0 \\
\hline De $50 \mathrm{mgr} .0$ à $70 \mathrm{mgr}$.par kilogramme & 3 & 6,7 \\
\hline 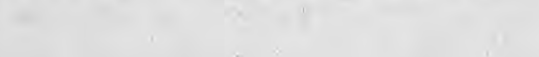 & $\overline{45}$ & 100,0 \\
\hline
\end{tabular}

Les teneurs sont pour la majeure partie comprises entre quelques milligrammes et $25 \mathrm{mgr}$. par kilogramme, avec toutefois $20 \%$ des beurres renfermant de 25 à $50 \mathrm{mgr}$. d'acétylméthylcarbinol.

En général, les beurres pauvres en diacétyle sont le plus souvent pauvres ou assez pauvres en acétylméthylcarbinol, et les beurres riches en diacétyle le plus souvent riches en son produit de réduction, mais ce n'est pas à beaucoup près une règle absolue.

C'est toutefois le beurre le plus riche en diacétyle (2 mgr. 4) qui nous a donné le plus d'acétylméthylcarbinol (69 mgr. 1).

Mais les teneurs en ce dernier élément n'ont à notre avis qu'un intérêt documentaire, pour la question qui nous préoccupe plus spécialement, car si l'on admet que le diacétyle normal des beurres puisse disparaître progressivement par évaporation ou par réduction, il ne semble pas que l'acétylméthylcarbinol qui l'accompagne puisse régénérer par oxydation autre chose que des traces de diacétyle. Un beurre que nous avions privé de son diacétyle par distillation n'en avait régénéré après 10 jours que $0 \mathrm{mgr}$. 08 environ.

Teneur des crèmes et du babeurre en diacétyle et en acétylméthylcarbinol. - Nous avons examiné une douzaine 
de crèmes et quelques babeurres (1) provenant des laiteries de la Manche et du Calvados; malheureusement onze d'entre elles ne peuvent être comparées aux beurres prélevés lo jour du passage de M. BARBAzAN ; les beurres provenaient des crèmes fermentées de la veille, dont il ne restait plus, et les crèmes prélevées étaient des crèmes fraîches du jour même.

Elles nous ont donné à l'analyse :

\begin{tabular}{|c|c|c|}
\hline Provenance & Diacétyle & $\begin{array}{c}\text { Acétylméthyl- } \\
\text { carbinol }\end{array}$ \\
\hline & (mgr.) & (mgr.) \\
\hline Laiterie Coopérative de Trébehou .... & 0,13 & 45,9 \\
\hline Laiterie Coopérative de Ducey . . . . . . . & 1,36 & 8,2 \\
\hline Laiterie Coopérative de Montebourg . . & 1,60 & 15,5 \\
\hline Laiterie Coop. de St-Sauveur-le-Vic. . . & 0,28 & ; \\
\hline Industrie laitière du Cotentin.......... & 0,64 & 1,9 \\
\hline Société du " Lait Gloria » . . . . . . . . & 0,08 & 2,8 \\
\hline Laiterie Coop. de Périers.......... . & 0,23 & \\
\hline Laiterie de Pont-Hébert ........ & 0,34 & 2,2 \\
\hline Laiterie Coop. de Ste-Mère-Eglise . . . & 1,23 & 44,8 \\
\hline Laiterie industr. de Chef-de-Pont ... & 0,80 & \\
\hline Laiterie Coop. de Bayeux (Vaucelles) . . & 2,03 & 10,7 \\
\hline
\end{tabular}

La plupart de ces crèmes n'ayant pas subi, en masse, une fermentation normale ne sont riches ni en diacétyle (sauf quatre) ni en acétylmétylcarbinol.

Il n'en est pas de même pour une crème du Calvados, provenant de la Laiterie de Villers-Bocage, et pour deux échantillons provenant de la Laiterie coopérative de Royville (S.-I.), qui nous étaient apportés en même temps que les beurres et babeurres purs non dilués d'eau, le jour même du barattage, et que l'on analysait immédiatement, ou au plus tard le lendemain matin, après séjour d'une nuit à la glacière.

Les crèmes sont sensiblement plus riches en diacétyle, et surtout en acétylméthylcarbinol que les beurres correspondants; mais ces deux corps passent surtout dans le babeurre; les doses d'acéthylearbinol que l'on y trouve sont même assez considérables.

A la Laiterie coopérative de Royville, les crèmes sortant des écrémeuses ne sont pas pasteurisées, ni ensemencées ; elles subissent seulement une fermentation naturelle de 24 heures, précédant le barattage.

(1) Les babeurres étaient tous fortement dilués d'eau, aussi nous laissons de côté Ies résultats trouvés. 


\begin{tabular}{|c|c|c|}
\hline Nature et provenance des échantillons & $\begin{array}{c}\text { Diacétyle } \\
\text { (mgr.) }\end{array}$ & $\begin{array}{c}\text { Acétylméthyl- } \\
\text { carbinol } \\
\text { (mgr.) }\end{array}$ \\
\hline \multicolumn{3}{|l|}{ Laiterie de Villers-Bocage : } \\
\hline Crème ........... & 1,10 & 102,1 \\
\hline Beurre & 0,60 & 19,8 \\
\hline \multicolumn{3}{|l|}{ Laiterie de Royville: } \\
\hline jer envoi ( crème .............. & 2,25 & 176,7 \\
\hline foring beurre. & 1,13 & 23,4 \\
\hline (babeurre ............... & 4,32 & 198,1 \\
\hline \multirow{3}{*}{$\begin{array}{l}2^{\mathrm{e}} \text { envoi } \\
23 \text { juin }\end{array}$} & 1,92 & 95,2 \\
\hline & 1,50 & 42,4 \\
\hline & 3,10 & 195,3 \\
\hline
\end{tabular}

Le partage du diacétyle et de l'acétylméthylcarbinol de la crème entre le beurre et le babeurre mériterait d'être étudié plus à fond, mais nous estimons qu'il devrait l'être à la laiterie même pour éliminer les causes de perte ou d'erreurs résultant du transport des échantillons et des délais avant l'analyse.

Que devient le diacétyle naturel des beurres? - Divers auteurs ont déjà établi que le diacétyle des beurres frais disparaît peu à peu sous diverses influences qui ne sont pas précisées.

Nous avons cherché à nous documenter personnellement sur ce point. Nous avons mis en réserve d'abord à la glacière, à $+6^{\circ}$, puis ensuite simplement dans une cave fraîche, une motte de beurre de la Laiterie de Royville, nous ayant fourni, le 24 juin, $1 \mathrm{mgr} .5$ de diacétyle par kilogramme.

Les différents dosages échelonnés donnèrent:

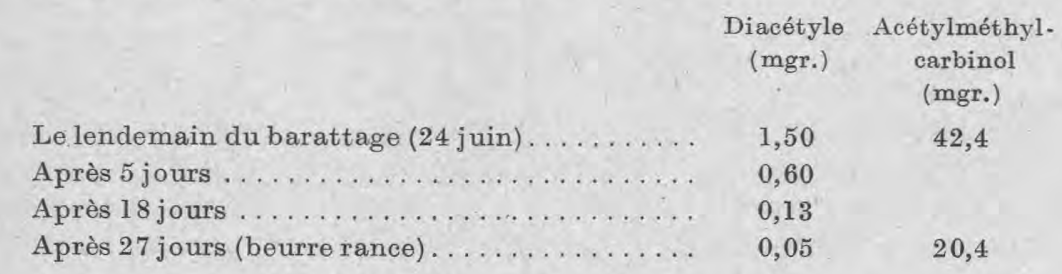

La teneur en diacétyle diminue done assez rapidement, l'acétylméthylcarbinol a diminué lui-même de moitié en 27 jours.

De même, le beurre du Concours beurrier de Rouen, qui dosait le lendemain du concours 1 mgr. 2 de diacétyle, n'en contenait plus, 
après 6 jours de conservation par temps chaud, que 0 mgr. 23 par kílogramme.

Cette constatation présente une assez grande importance au point de vue des analyses effectuées par les laboratoires du Service de la Répression des Fraudes, qui ne reçoivent souvent les échantillons qu'une huitaine de jours après leur prélèvement, et plus encore au point de vue des expertises, car les experts ne sont guère mis en possession des échantillons suspectés de fraude, qu'après un mois à un mois et demi.

Que peut-il rester alors du diacétyle naturel des beurres ou de celui qui est surajouté ? Ce dernier agit d'ailleurs, d'après divers expérimentateurs (TAPERnoux, Pien, etc...), comme un catalyseur d'oxydation, avec rancissement plus ou moins rapide de la matière grasse. $\mathrm{Il} \mathrm{y}$ a encore là des points à préciser.

Résumé et principales conclusions. - De nos recherches et analyses, il semble que nous puissions tirer les conclusions suivantes:

$1^{0}$ Les beurres frais normands renfernient normalement une petite quantité de diacétyle, qui est le plus souvent comprise entre 0 mgr. 05 et 0 mgr. 5 par kilogramme; mais certains beurres peuvent en contenir de $1 \mathrm{mgr}$. à $1 \mathrm{mgr}$. 5, et exceptionnellercent, semblet-il, jusqu'à $2 \mathrm{mgr}$. 5 .

$2^{\circ}$ Le diacétyle normal contenu dans les beurres frais disparaît assez rapidement sous des influences encore mal élucidées : après 15 i 18 jours, il peut n'en rester qu'un dixième de ce qui existait le jour ou le lendemain du barattage. Ceci est très important du point de vue des expertises.

$3^{\circ}$ Les doses d'acétylmóthylearbinol sont sensiblement plus fortes que les doses de diacétyle, et le plus souvent comprises entre 10 et 30 mgr. par kilogramme ; elles ne paraissent pas pouvoir régénérer une proportion sensible de diacétyle.

$4^{\text {n }}$ Le diacétyle et l'acétylméthylcarbinol des erèmes fermentées se retrouvent en majorité dans le babeurre, principalement l'acétylméthylcarbinol; le beurre n'en retient qu'une fraction, plus élevée généralement pour le diacétyle.

C'ertains points mériteraient d'être approfondis, mais ne peuvent l'être sérieusement, à notre avis, que par un expérimentateur séjournant dans une laiterie pourvue d'un laboratoire. 